\title{
Comments on the hierarchy problem in effective theories
}

\section{Archil Kobakhidze and Kristian L. McDonald}

ARC Centre of Excellence for Particle Physics at the Terascale, School of Physics, The University of Sydney, NSW 2006 Australia

E-mail: archilk@physics.usyd.edu.au, klmcd@physics.usyd.edu.au

ABSTRACT: We discuss aspects of the hierarchy problem in effective theories with light scalars and a large, physical ultraviolet (UV) cutoff. We make two main points: (1) The (naive) fine-tuning observed in an effective theory does not automatically imply that the UV completion is fine tuned. Instead, it gives a type of upper bound on the severity of the actual tuning in the UV completion; the actual tuning can be less severe than the naive tuning or even non-existent. (2) Within an effective theory, there appear to be two types of parameter relations that can alleviate the sensitivity of the scalar mass to the cutoff - relationships among dimensionless couplings or relationships among dimensionful parameters. Supersymmetric models provide symmetry-motivated examples of the former, while scale-invariant models give symmetry-motivated examples of the latter.

Keywords: Beyond Standard Model, Space-Time Symmetries, Standard Model

ARXIV EPRINT: 1404.5823 


\section{Contents}

1 Introduction 1

2 Naive-tuning versus actual-tuning $\quad 2$

3 Removing the cutoff via parameter relations 5

$\begin{array}{lll}3.1 & \text { Relationships among dimensionless couplings } & 6\end{array}$

$\begin{array}{lll}3.2 & \text { Relationships among dimensionful couplings } & 8\end{array}$

4 Conclusion

\section{Introduction}

The hierarchy problem [1-3] has driven much research into physics beyond the Standard Model (SM) in recent decades. When taken as an effective theory with a large cutoff, it is difficult to understand how the Higgs boson remains light relative to the large cutoff effects. With the experimental discovery of the Higgs boson at the LHC, the desire to understand how scalars can remain light, despite large cutoff-dependent corrections, has gained urgency in recent years [4-9].

In this work we discuss aspects of the hierarchy problem in effective theories with light scalars and a large physical ultraviolet (UV) cutoff. We discuss two main points. Firstly, we note that the (naive) fine-tuning found in an effective theory does not automatically imply that the UV completion is fine tuned. Rather, it gives a sort of upper bound on the severity of the actual tuning present in the underlying UV completion; the actual tuning due to the new physics at the cutoff scale can be less severe than the naive tuning or even non-existent. Secondly, we note that within an effective theory there appear to be two types of parameter relations that can alleviate the sensitivity of the scalar mass to the cutoff; a relationship among dimensionless couplings á la the Veltman condition, or a relationship among dimensionful parameters. Supersymmetric (SUSY) models give symmetry-motivated examples in which a technically-natural Veltman-like condition arises, while scale-invariant models are symmetry-motivated examples where relations between dimensionful parameters are expected.

In essence, these two points pertain to the two different aspects of the hierarchy problem, namely the naturalness of new particle thresholds and the tuning associated with "pure cutoff" effects. If the UV physics takes the form of new thresholds, or behaves similar to such, the naive tuning can overestimate the actual tuning. This has implications for model-building approaches to beyond-SM physics [7, 9]. However, if the cutoff accurately represents the behaviour of the UV physics, one expects a natural theory to shield the infrared (IR) sector. These points are elaborated within. 
The layout of this paper is as follows. In section 2 we discuss the naive tuning in an effective theory and consider a simple example where the actual tuning in the UV completion can be less severe. In section 3 we consider simple relationships among parameters in an effective theory that can shield the IR sector from cutoff effects. Conclusions are drawn in section 4 .

\section{$2 \quad$ Naive-tuning versus actual-tuning}

Consider the effective theory for a self-interacting scalar field $S$, with Lagrangian

$$
\mathcal{L}_{S}^{\Lambda}=\partial^{\mu} S^{*} \partial_{\mu} S-\bar{m}_{S}^{2}(\Lambda)|S|^{2}-\lambda_{S}|S|^{4}+\ldots
$$

The parameters depend on the cutoff scale $\Lambda$, and the dots denote non-renormalizable irrelevant IR operators with mass-dimension $d>4$, suppressed by factors of $\Lambda^{4-d}$. Calculating the one-loop corrected mass in the effective theory gives ${ }^{1}$

$$
m_{S}^{2}=\bar{m}_{S}^{2}(\Lambda)+\delta m_{\Lambda}^{2} \equiv \bar{m}_{S}^{2}(\Lambda)+\lambda_{S}\left\{\Lambda^{2}+\bar{m}_{S}^{2}(\Lambda) \log \left(\bar{m}_{S}^{2}(\Lambda) / \Lambda^{2}\right)\right\}
$$

For large values of $\Lambda$, the theory appears to have a hierarchy problem, with small values of $m_{S}^{2} \ll \Lambda^{2}$ requiring a fine-tuning between $\bar{m}_{S}(\Lambda)$ and $\Lambda$. The effective theory is said to be fine-tuned because the origin of this cancellation is understood within the effective theory.

It is important to distinguish two qualitatively different contexts in which eq. (2.2) is interpreted. In the first case, $\Lambda$ is merely as a tool to regularize the divergent loop-integral. Then, both $\Lambda$ and the $\Lambda$-dependent bare parameters are regarded as unphysical quantities. In fact, one must take the limit $\Lambda \rightarrow \infty$ and remove all divergences by renormalizing the unphysical bare masses and couplings, leaving finite physical parameters. From the symmetry perspective, a theory with an unphysical cut-off possesses a scale invariance that is softly broken by the explicit mass terms and the logarithmic quantum corrections [10]. The softness of the breaking is reflected in the infrared fixed-point structure of the mass RGEs [11, 12].

The second interpretation of eq. (2.2), of interest here, applies when the cut-off $\Lambda$ is physical and therefore associated with a new scale in the UV-completion of the effective theory. In this case the effective theory has a hierarchy problem [1-3], and the cut-off $\Lambda$, as well as the $\Lambda$-dependent bare parameters and the set of irrelevant operators, fully encode information about the UV theory. Absent knowledge of the underlying theory, one cannot renormalize away the $\Lambda$-dependence. We refer to the fine-tuning associated with this hierarchy problem as the "naive fine-tuning of the effective theory," or more simply as "the naive tuning." For a given fixed value of $\lambda_{S}=\mathcal{O}(1)$ (for example), this naive tuning requires two parameters of $\mathcal{O}\left(\Lambda^{2}\right)$ to cancel out at a precision of $\mathcal{O}\left(m_{S}^{2} / \Lambda^{2}\right)$. With $m_{S}^{2} \ll \Lambda^{2}$ this tuning is severe.

One can phrase the hierarchy problem of eq. (2.2) in the following way. Consider values of the parameters in the effective theory that generate some fixed value for the scalar mass

\footnotetext{
${ }^{1}$ We suppress numerical loop-factors in this section.
} 
$m_{S}^{2}$. Now shift the bare mass $\bar{m}_{S}^{2}(\Lambda)$ as follows:

$$
\bar{m}_{S}^{2}(\Lambda) \rightarrow \bar{m}_{S}^{2}(\Lambda)+\delta \bar{m}_{S}^{2}(\Lambda), \quad \text { with } \quad \frac{\delta \bar{m}_{S}^{2}(\Lambda)}{\bar{m}_{S}^{2}(\Lambda)} \lesssim \mathcal{O}(1) .
$$

This will, in general, induce a shift in the scalar mass:

$$
m_{S}^{2} \rightarrow m_{S}^{2}+\delta m_{S}^{2} .
$$

The effective-theory has a hierarchy problem if $\delta m_{S}^{2} / m_{S}^{2} \gg \mathcal{O}(1)$ - i.e. if small changes in the bare mass create a large change in the scalar mass. For generic couplings, an effective theory with $m_{S}^{2} \ll \Lambda^{2}$ is expected to have a hierarchy problem. One must fine-tune $\bar{m}_{S}^{2}(\Lambda) \simeq \mathcal{O}\left(\Lambda^{2}\right)$ against the $\Lambda^{2}$-term to enable $m_{S}^{2} \ll \Lambda^{2}$. Thus, shifts of $\delta \bar{m}_{S}^{2}(\Lambda) / \bar{m}_{S}^{2}(\Lambda)=\mathcal{O}(1)$ give $\delta \bar{m}_{S}^{2}(\Lambda)=\mathcal{O}\left(\Lambda^{2}\right)$, which in turn gives

$$
m_{S}^{2} \rightarrow m_{S}^{2}+\mathcal{O}\left(\Lambda^{2}\right) \gg m_{S}^{2}
$$

The hierarchy problem manifests through this extreme sensitivity of the physical scalar mass to small changes in the effective-theory mass-parameter.

With knowledge of the UV completion for the theory $\mathcal{L}_{S}^{\Lambda}$, one can investigate the origin of the naive tuning by calculating the "actual tuning" in the UV completion. One would like to know if the naive tuning accurately encodes the actual tuning. Our main point in this section is that the actual tuning can be less severe than the naive tuning and in some cases may even be absent. One should think of the naive tuning as representing a worst case scenario for the severity of the tuning associated with the new physics at the scale $\Lambda$. We demonstrate this with a simple example.

The scale $\Lambda$ is assumed physical, in the sense that new physics appears at this scale. There are, in principle, two classes of UV completions that one could consider. In one class, the scalar $S$ persists as a physical degree of freedom beyond the scale $\Lambda$, and the new physics takes the form of additional degrees of freedom with mass $M_{H} \sim \Lambda$. A typical example occurs when the theory is UV completed by a new heavy scalar, $H$. If the two scalars couple via a quartic interaction, $\lambda_{\text {mix }} S^{2} H^{2}$, the UV completion generates a mass correction for the light scalar of the form

$$
\delta m_{S, H}^{2}=\lambda_{\operatorname{mix}} M_{H}^{2} \log \left(M_{H}^{2} / \mu^{2}\right),
$$

where $\mu$ denotes a renormalization scale in the UV completion. ${ }^{2}$ With regard to fine-tuning due to $H$, one can differentiate three cases:

- For $\lambda_{\text {mix }} \sim \mathcal{O}(1)$, there is a hierarchy problem and a light scalar with $m_{S} \ll M_{H}$ requires a fine-tuned UV completion. The required tuning is at the level of $\mathcal{O}\left(m_{S}^{2} / \Lambda^{2}\right)$, occurring between quantities of $\mathcal{O}\left(\Lambda^{2}\right)$. In this case the actual tuning agrees with the naive tuning of the IR observer. An analogous example is the UV completion of the SM by a renormalizable Grand Unified Theory, with unification scale $M_{\mathrm{GUT}} \sim \Lambda \gg 10^{2} \mathrm{GeV}$.

\footnotetext{
${ }^{2}$ For our purpose in this section it suffices to take the cutoff for the UV theory merely as a regulator. We further discuss cutoff effects in the next section.
} 
- For $m_{S}^{2} / M_{H}^{2} \ll \lambda_{\text {mix }} \ll 1$, there is still a hierarchy problem and the UV completion remains fine-tuned. However, now the actual tuning is at the level of $\mathcal{O}\left(m_{S}^{2} /\left[\lambda_{\text {mix }} M_{H}^{2}\right]\right)$, between quantities of $\mathcal{O}\left(\lambda_{\text {mix }} M_{H}^{2}\right) \ll \Lambda^{2}$. The actual tuning due to the physics at $\Lambda$ is less severe than the naive tuning. An analogous example is the UV completion of the SM by heavy right-handed neutrinos, to generate neutrino masses via the seesaw mechanism, with heavy Majorana masses in the range ${ }^{3}$ $10^{12} \gg\left(M_{R} / \mathrm{GeV}\right) \gg 10^{7}$. Radiative corrections to the Higgs mass then exceed the observed value, necessitating a tuning between parameters of $\mathcal{O}\left(y_{\nu}^{2} M_{R}^{2}\right) \ll \Lambda^{2}$, where $y_{\nu}^{2} \Leftrightarrow \lambda_{\text {mix }}$ is the Dirac Yukawa-coupling.

- For $\lambda_{\text {mix }} \lesssim m_{S}^{2} / M_{H}^{2}$, there is no hierarchy problem. The loop-correction does not exceed the physical scalar mass and the theory is technically natural. This case is contrary to the naive expectation of the IR observer; the naively-tuned effectivetheory possesses a technically-natural UV completion. An analogous example is the UV completion of the SM by heavy right-handed neutrinos, giving neutrino mass via the seesaw mechanism, with heavy Majorana masses $\left(M_{R} / \mathrm{GeV}\right) \lesssim 10^{7}[9,13-15]$. Radiative corrections to the Higgs mass are less than the observed value, and the theory is devoid of tuning. Another example is the invisible axion model, which can solve the strong CP-problem and give a dark matter candidate $[7,9,16]$.

Note that the second and third cases are only possible if values of $\lambda_{\operatorname{mix}} \ll 1$ are technically natural. This is true if, e.g., the limit $\lambda_{\operatorname{mix}} \rightarrow 0$ gives an enhanced Poincaré symmetry [9], as occurs here. This example shows that the naive tuning in an effective theory can be more severe than the actual tuning due to the physics at the scale $\Lambda$.

In the other class of UV completions for $\mathcal{L}_{S}^{\Lambda}$, there is a transition to a new theory at the scale $\Lambda$, such that the degrees of freedom are different and the scalar $S$ ceases to exist. For example, this occurs if $S$ is a low-energy composite object ${ }^{4}$ and the transition to more-fundamental "quarks" occurs at $\Lambda$, and similarly if $\Lambda$ is a minimal length-scale in the UV theory ${ }^{5}$ or a type of non-locality scale such as in string theory. In these examples the actual tuning required in the UV completion is expected to be as severe as the naive tuning.

Note that an IR observer cannot differentiate between these two classes of UV completions, absent information about the UV physics. When faced with a naively-tuned effective theory an IR observer can, at best, conclude that the underlying UV completion may be fine tuned, due to new physics at the scale $\Lambda$. The naive tuning provides a type of upper bound for the severity of the actual tuning in the UV completion as a result of the new physics at the scale $\Lambda$; the actual tuning in the completion can be less severe or in some cases even non-existent.

\footnotetext{
${ }^{3}$ The upper bound results from the standard seesaw expression, assuming $\mathcal{O}(1)$ Dirac Yukawa-couplings and that the SM neutrinos have masses $m_{\nu} \sim 0.1 \mathrm{eV}$.

${ }^{4}$ In general, one would expect more IR composite states than a single scalar for this case.

${ }^{5}$ Analogous to the role played by the inter-atomic spacing when describing spin-correlation functions of a magnetic system by an effective scalar field theory.
} 
The physical origin of the fine-tuning in the two classes of UV completions is distinct. The $\Lambda^{2}$-term in (2.2) encodes the mass correction to the IR scalar $S$, due to the UV (Euclidean) momentum modes with $\left|p_{\mathrm{E}}\right| \sim \Lambda$ for the IR degree of freedom. In the first class of models, where heavy new physics with mass $M_{H} \sim \Lambda$ appears, the origin of the actual tuning differs from the source of the naive tuning. The actual tuning, if present, is due to mass corrections from the heavy UV physics, while the naive tuning results from UV momentum modes for the IR fields. These two effects have distinct physical meanings and this is why the naive tuning and the actual tuning can differ. One should think of the naive tuning as being a proxy for the actual tuning - the existence of a naive tuning in the IR theory indicates that the UV completion may contain an actual tuning. Note that, from the perspective of the UV theory, there is nothing special about modes with $\left|p_{\mathrm{E}}\right| \sim \Lambda$ for the IR scalar; these just happen to have the same momentum as the new physics scale.

In the case where $S$ does not persist in the UV, the momentum modes with $\left|p_{\mathrm{E}}\right| \sim$ $\Lambda$ for the IR scalar $S$ are "special" in the sense that modes with $\left|p_{\mathrm{E}}\right|>\Lambda$ simply do not exist. Now the $\Lambda^{2}$-term has a clear physical meaning and one understands why the modes $\left|p_{\mathrm{E}}\right| \sim \Lambda$ would give a mass correction of greater physical significance than modes with, e.g., $\left|p_{\mathrm{E}}\right| \sim \Lambda^{\prime} \ll \Lambda$. In this case there is no obvious reason why the bare mass and the cutoff should cancel-out so precisely; the natural value for the scalar mass is expected to be $m_{S} \sim \Lambda$. Said differently, the naive tuning is expected to well-approximate the actual-tuning.

Finally, we note that there are examples where a tree-level tuning of $\mathcal{O}\left(m_{S}^{2} / \Lambda^{2}\right)$ occurs in the UV. Such tunings can be slightly more severe than the tuning in the effective theory due to the loop suppression of the latter. However, taking the naive tuning as being $\mathcal{O}\left(m_{S}^{2} / \Lambda^{2}\right)$, as done here, means the actual tuning is not more severe than the naive tuning in these cases.

\section{Removing the cutoff via parameter relations}

We now turn to a different aspect of the hierarchy problem. Having focused mainly on new threshold effects in the preceding, we now focus on the $\Lambda^{2}$-term, assuming that it encodes a real physical effect that must be dealt with, for the theory to be natural. We consider the simplest possibilities for alleviating the cutoff sensitivity within the effective theory, namely by parameter relations that shield the light scalar mass from cutoff effects. To discuss this matter it is helpful to consider a more detailed IR sector.

Consider the effective theory for a system of real scalars $S=\left(s_{1}, s_{2}, \ldots\right)^{\mathrm{T}}$, gauge fields $V$, and Weyl fermions $F$, assumed valid up to a large UV cut-off $\Lambda$, which is understood in the Wilsonian sense. If present, a hierarchy problem would manifest in the relevant operators $S_{a} S_{b}$ that appear in the quantum-corrected effective action. The coefficients of these operators are given by the non-derivative part of the 2-point functions $\Gamma_{a b}^{(2)}$. In the 1-loop approximation they are:

$$
\left(m_{S}^{2}(\Lambda, \mu)\right)_{a b}=\left(\bar{m}_{S}^{2}(\Lambda)\right)_{a b}+\sum_{A=S, V, F}(-1)^{2 J_{A}}\left(2 J_{A}+1\right) \frac{\left(g_{A}\right)_{a b c d}}{16 \pi^{2}}\left[\Lambda^{2} \delta_{c d}-\left(\bar{m}_{A}^{2}(\Lambda)\right)_{c d} \ln \frac{\Lambda^{2}}{\mu^{2}}\right]
$$


where $\bar{m}_{A}^{2}(\Lambda)$ is the effective bare-mass for the field $A$ of spin $J_{A}$, and $\mu$ is an arbitrary renormalization scale, $\left|m_{A}\right|<\mu<\Lambda$. Here $g_{A}$ denotes the matrix of dimensionless couplings between the field $A$ and the scalars $S$, defined through the interaction terms as

$$
\frac{1}{4 !}\left(g_{S}\right)_{a b c d} S_{a} S_{b} S_{c} S_{d}, \quad \frac{1}{2}\left(g_{V}\right)_{a b c d} V_{a} V_{b} S_{c} S_{d} \quad \text { and } \quad\left(y_{F}\right)_{a b c} \bar{F}_{a} F_{b} S_{c}
$$

where $\left(y_{F}\right)_{a b k}\left(y_{F}\right)_{c d k}=\left(g_{F}\right)_{a b c d}$. As before, for light values of $m_{S}^{2} \ll \Lambda^{2}$, the effective theory has a hierarchy problem.

How can one remove this sensitivity to the cutoff? From the perspective of the effective theory, there appear to be two types of relationships that could remove the cutoff sensitivity in eq. (3.1). One could consider a relationship among dimensionless couplings that cancels the $\Lambda^{2}$-term, or a relationship among dimensionful quantities that relates the bare mass to the UV scale. For such a relationship to provide a viable explanation (i.e. not transfer the tuning to a different sector), it should be motivated by a symmetry. We discuss these two cases in turn.

\subsection{Relationships among dimensionless couplings}

First consider the case where the $\Lambda^{2}$-dependence is removed by a relationship among dimensionless couplings. We discuss two examples, differentiated by the absence/presence of an underlying symmetry.

The Veltman condition. The $\Lambda^{2}$-term in eq. (3.1) disappears if the dimensionless couplings in the theory are related:

$$
\sum_{A=S, V, F}(-1)^{2 J_{A}}\left(2 J_{A}+1\right)\left(g_{A}\right)_{a b c c}=0 .
$$

This possibility was introduced by Veltman [17], and studied prior-to (after) the Higgs discovery in refs. [18-20] ([21-25]). In an effective theory satisfying eq. (3.3) an IR observer can experimentally measure the couplings and determine that eq. (3.3) holds. When applied to the SM, this can be converted into a mass relation: ${ }^{6}$

$$
m_{h}^{2}+2 m_{W}^{2}+m_{Z}^{2}-4 m_{t}^{2}=0,
$$

giving $m_{h} \sim 300 \mathrm{GeV}$, in conflict with the data. However, a variant of this relation might be satisfied in the UV, with additional beyond-SM fields also participating [21-25]. If the Veltman condition is realized, the $\Lambda^{2}$-term in eq. (3.1) cancels out and the bare Higgs mass is similar to the observed mass, $m_{h}^{2} / \bar{m}_{h}^{2}(\Lambda)=\mathcal{O}(1)$. Consequently, small changes to the bare mass give small changes to the Higgs mass, and the theory appears natural. However, the (generalized) Veltman condition is not motivated by any symmetry. From the perspective of the effective theory, one cannot understand why such a relationship exists, nor how it remains radiatively stable. If a Veltman-like relation holds, the hierarchy

\footnotetext{
${ }^{6}$ In general, models with related couplings do not predict a mass relation. This feature of the SM arises because the masses for all fundamental particles are set by the Higgs vacuum value. More general theories can have vector-like fermions or scalars with explicit masses not related to symmetry breaking.
} 
problem is revealed by looking at small shifts in individual dimensionless couplings. Under a small shift $g_{A} \rightarrow g_{A}+\delta g_{A}$ for a particular coupling $g_{A}$, the cancellation of the $\Lambda^{2}$-term ceases to function and the need for fine-tuning is manifest. Thus, the hierarchy problem of the effective theory is not resolved; one has simply exchanged a tuning among massparameters for a tuning amongst couplings.

Supersymmetry. Models with exact SUSY possess an equal number of bosonic and fermionic degrees of freedom (i.e., $\left.\sum_{A=S, V, F}(-1)^{2 J_{A}}\left(2 J_{A}+1\right)=0\right)$. Furthermore, SUSY forces relationships among dimensionless coupling constants, and requires multiplets to be mass-degenerate, namely

$$
\sum_{A=S, V, F}(-1)^{2 J_{A}}\left(2 J_{A}+1\right)\left(g_{A}\right)_{a b c c}=0
$$

and

$$
\sum_{A=S, V, F}(-1)^{2 J_{A}}\left(2 J_{A}+1\right)\left(g_{A}\right)_{a b c d}\left(m_{A}\right)_{c d}^{2}=0 .
$$

These equations reflect the perturbative non-renormalization theorem [26], according to which only wavefunction renormalization is required in $\mathrm{N}=1 \mathrm{SUSY}$ theories. Eq. (3.5) solves the hierarchy problem because it forces the coefficient of the $\Lambda^{2}$-term to vanish in eq. (3.1). Note the similarity with the Veltman condition; it is clear that SUSY models can be thought of as symmetry-motivated examples where a Veltman-like condition is automatically achieved.

In realistic applications, effective theories describing supersymmetric extensions of the SM cannot possess exact SUSY. However, eq. (3.5) also holds in softly-broken SUSY theories, since the soft SUSY-breaking terms are dimensionful parameters that do not appear in (3.5). On the other hand, eq. (3.6) is modified because the soft-breaking terms lift the fermion-boson mass degeneracy, and set the SUSY-breaking scale,

$$
\sum_{A=S, V, F}(-1)^{2 J_{A}}\left(2 J_{A}+1\right)\left(g_{A}\right)_{a b c d}\left(m_{A}\right)_{c d}^{2} \sim M_{\mathrm{SUSY}}^{2} .
$$

Then, provided $M_{\text {SUSY }} \ll \Lambda$, light scalars with mass $m_{S}^{2}=\mathcal{O}\left(M_{\text {SUSY }}^{2}\right)$ are technically natural in softly-broken SUSY models. In terms of the bare scalar mass, this corresponds to $\bar{m}_{S}^{2}(\Lambda) \lesssim \mathcal{O}\left(M_{\text {SUSY }}\right)$, so a small shift induces the change $\delta m_{S}^{2} \lesssim \mathcal{O}\left(M_{\text {SUSY }}^{2}\right)$ in the physical mass, manifesting naturalness.

If SUSY is broken in the UV completion, then presumably the fermion-boson mass degeneracy is broken by large $\mathcal{O}(\Lambda)$ effects in some heavy (hidden) sector of the theory. The light (visible) sector must couple to the heavy sector with sufficiently weak couplings to enable $m_{S}^{2} \sim M_{\text {SUSY }}^{2} \ll \Lambda^{2}$. This is what happens in most of the realistic particle physics models, where SUSY is spontaneously broken at a high energy scale in a heavy hidden sector, and feebly communicated to the visible sector.

In the case of hard SUSY-breaking, eq. (3.5) is also violated and the SUSY-breaking scale is set by

$$
\sum_{A=S, V, F}(-1)^{2 J_{A}}\left(2 J_{A}+1\right)\left(g_{A}\right)_{a b c c} \Lambda^{2} \sim M_{\mathrm{SUSY}}^{2}
$$


Light scalars can only be accommodated if the hard SUSY-breaking dimensionless couplings are sufficiently small, i.e. the relation (3.5) is satisfied with sufficient accuracy. We stress that hard SUSY-breaking also allows technically-natural light scalars, as the limit when eq. (3.5) is strictly satisfied corresponds to an increased symmetry in the theory. In fact, small, hard SUSY-breaking terms frequently appear in SUSY models without causing problems.

Recall that, although the $\Lambda^{2}$-term canceled out in models with a Veltman condition, the hierarchy problem manifested under small shifts to the dimensionless couplings, $g_{A} \rightarrow$ $g_{A}+\delta g_{A}$. SUSY cures this problem by demanding that the shift $g_{A} \rightarrow g_{A}+\delta g_{A}$ is accompanied by complimentary shifts $g_{A}^{\prime} \rightarrow g_{A}^{\prime}+\delta g_{A}^{\prime}$ in any couplings related to $g_{A}$ through SUSY. One must enforce a relationship among dimensionless couplings to ensure that eq. (3.5) is satisfied. To vary a single coupling without varying the SUSY-related couplings would amount to a departure from the physical content of the theory. This symmetry-motivated origin for the coupling-relation ensures its stability under radiative corrections and tells us the couplings are likely born in some related way.

To summarize, in the presence of two (or more) sectors with hierarchically different masses, SUSY can ensure stability of the hierarchy if it is broken softly with all the softbreaking mass parameters being of order $M_{\text {SUSY }} \ll \Lambda$, as in softly-broken SUSY GUTs. In other cases, the heavy hidden sector should couple to the light visible sector very weakly. The latter case may or may not be technically natural, depending on details of the model [9].

\subsection{Relationships among dimensionful couplings}

SUSY models and the Veltman-condition both cancel out the quadratic divergences via a relationship among dimensionless couplings. The other possibility is that the sensitivity to the cutoff in eq. (3.1) is alleviated by a relationship among dimensionful parameters in the effective theory. Indeed, the hierarchy problem of the SM manifests as a tuning between the bare scalar-mass and the UV scale - this sensitivity between the only two dimensionful parameters of the effective theory may suggest a deeper connection.

Relationship without symmetry. In analogy with the Veltman condition, one can imagine a UV completion that triggers a relationship between the dimensionful parameters in the effective theory. However, for arbitrary relations among dimensionful parameters, that are not motivated by a symmetry, one cannot understand the origin of the relation within the effective theory. The relation is therefore equivalent to a tuning. For example, if the UV completion triggered the relation

$$
\left(\bar{m}_{S}^{2}(\Lambda)\right)_{a b}+\sum_{A=S, V, F}(-1)^{2 J_{A}}\left(2 J_{A}+1\right) \frac{1}{16 \pi^{2}}\left(g_{A}\right)_{a b c c} \Lambda^{2}=M_{a b}^{2},
$$

for some fixed $M_{a b}^{2} \ll \Lambda^{2}$, such that the bare scalar masses and the UV scale are related, this would "remove" the quadratic divergence. However, the IR observer could not distinguish this from a fine-tuning - this relation is the standard expression for the fine-tuning! It requires a large bare mass, $\bar{m}_{S}^{2}(\Lambda) \sim \Lambda^{2}$, that precisely cancels the cutoff effects to allow a light scalar. As with the Veltman condition, one cannot understand how such a 
relationship remains stable under radiative corrections in the effective theory framework, so the tuning persists. This is evidenced by the fact that a small shift in the bare-mass, $\delta \bar{m}_{S}^{2}(\Lambda) / \bar{m}_{S}^{2}(\Lambda)=\mathcal{O}(1)$, corresponds to $\delta \bar{m}_{S}^{2}(\Lambda)=\mathcal{O}\left(\Lambda^{2}\right)$, giving a large shift in the physical mass that drags it up to the UV scale. Note that the dimensionless couplings measured by the IR observer do not satisfy eq. (3.3) in this case, revealing that the $\Lambda^{2}$ term does not vanish and that the bare mass must be large.

Scale invariance. Within an effective theory, scale-invariance appears to be badly broken. In addition to the bare masses $\bar{m}_{A}(\Lambda)$, and the logarithmic quantum-anomalous terms, which break scale-invariance softly, one encounters hard-breaking relevant operators $\sim \Lambda^{2}$, which introduce the quadratic sensitivity of the light masses to the cut-off scale. Nevertheless, the effective theory can describe an underlying UV theory that maintains scale invariance, at least at the classical level. The renormalized mass terms computed (within the perturbative framework) in a scale-invariant theory are necessarily zero because all bare mass terms are absent as a result of the scale invariance [27]. On the other hand, the mass terms computed in a scale-invariant UV theory should match the corresponding mass terms $m_{S}^{2}(\Lambda, \mu)$, computed in the low-energy effective theory, at the matching scale defined by the effective theory cut-off, $\mu=\Lambda[28] .{ }^{7}$ It follows from eq. (3.1) that the IR effective theory for such UV theories automatically satisfies the relation,

$$
\left(\bar{m}_{S}^{2}(\Lambda)\right)_{a b}+\sum_{A=S, V, F}(-1)^{2 J_{A}}\left(2 J_{A}+1\right) \frac{1}{16 \pi^{2}}\left(g_{A}\right)_{a b c c} \Lambda^{2}=0,
$$

ensuring the effective theory accurately matches the UV theory at the matching scale $\Lambda$. Thus, only a logarithmic sensitivity to the UV scale remains:

$$
\left(m_{S}^{2}(\Lambda, \mu)\right)_{a b}=\sum_{A=S, V, F}(-1)^{2 J_{A}}\left(2 J_{A}+1\right) \frac{1}{16 \pi^{2}}\left(g_{A}\right)_{a b c d}\left(m_{A}^{2}\right)_{c d} \ln \frac{\Lambda^{2}}{\mu^{2}} .
$$

It is important to emphasize that although classical scale-invariance is broken by quantum effects (the trace anomaly) this only introduces a logarithmic scale dependence, not hard $\Lambda^{2}$ terms. Thus, once the symmetry structure of the UV theory is understood, the apparent sensitivity of the scalar mass to the effective-theory cutoff is removed, leaving the logarithmic scale-dependence mandated by the trace anomaly.

Eq. (3.10) is a concrete example of a symmetry-motivated relationship among dimensionful parameters that removes the quadratic cutoff dependence of the scalar mass. In the previous section, the hierarchy problem in the effective theory was revealed by varying the bare mass while keeping the UV scale and the dimensionless couplings fixed; small changes in the bare mass induced large changes in the physical mass. However, in a scale-invariant theory, a shift made to the bare mass while keeping the other parameters fixed amounts to a departure from the physical content of the UV theory. That is, if the UV and IR scales are born in some common way, due to an underlying scale invariance, the shift

$$
\bar{m}_{S}^{2}(\Lambda) \rightarrow \bar{m}_{S}^{2}(\Lambda)+\delta \bar{m}_{S}^{2}(\Lambda), \quad \text { with } \quad \frac{\delta \bar{m}_{S}^{2}(\Lambda)}{\bar{m}_{S}^{2}(\Lambda)} \lesssim \mathcal{O}(1),
$$

\footnotetext{
${ }^{7} \mathrm{~A}$ number of works have studied scale-invariance in relation to the hierarchy problem [29-71], and discussion of quadratic divergences appears in refs. [72, 73].
} 
also requires a shift

$$
\Lambda^{2} \rightarrow \Lambda^{2}+\delta \Lambda^{2}, \quad \text { with } \quad \frac{\delta \Lambda^{2}}{\Lambda^{2}} \lesssim \mathcal{O}(1),
$$

to ensure an accurate low-energy effective-theory description. If the couplings are held fixed, a small change in the bare mass of the form (3.12) must come partnered with a small change in the UV scale of the form (3.13) to ensure that eq. (3.10) holds. More generally, the shift in the bare mass should be partnered with a compensating shift in the couplings and/or the UV-scale to ensure they remain related by eq. (3.10). Only then does the effective theory accurately encode the relationship between the two scales that is inherent in the parent theory.

Note that the soft-breaking masses $\bar{m}_{A}$ in scale invariant theories emerge from the spontaneous breaking of scale invariance, the mechanism known as dimensional transmutation [27]. Thus, for a single source of symmetry breaking, all such masses are proportional to the scale of this breaking, $M_{\mathrm{SI}}$. As with SUSY models, we again encounter two distinct possibilities: (i) $M_{\mathrm{SI}} \ll \Lambda$ and all the dimensionless couplings, and hence masses, are of the same order of magnitude [30,31], or (ii) $M_{\mathrm{SI}} \sim \Lambda$ and a hierarchy of masses exists due to a hierarchy among dimensionless coupling-constants; that is, hierarchically separated sectors of the theory interact sufficiently weakly to preserve the hierarchical scales $[9,32,33]{ }^{8}$

Finally, we note the similarity between the SUSY and scale-invariant narratives. If the SM is an effective theory that is UV completed by the MSSM, new symmetry-motivated degrees of freedom appear at the cutoff scale to enable a Veltman-like condition among dimensionless couplings. This protects the weak scale from quadratic divergences. In the scale-invariant case, a new scale appears in the UV which enables a symmetry-motivated relationship among dimensionful parameters, ensuring the IR scale of the effective theory is related to the UV scale. Quantization of course breaks scale-invariance softly via $\ln \Lambda$ operators, but this only affects marginal, $d=4$ operators in the low-energy effective theory.

\section{Conclusion}

In this work we discussed aspects of the hierarchy problem in effective theories with a light scalar. We sought to make two main points, namely: (1) The naive tuning in an effective theory can be more severe than the actual tuning in the UV completion; the naive tuning gives a type of upper bound for the severity of the actual tuning associated with the new physics at the cutoff scale. (2) There appear to be two classes of parameter relations that can alleviate the quadratic cutoff-dependence in an effective theory; relations among dimensionless couplings (of the Veltman type), which ensure that the quadratic divergences cancel out, or relations among dimensionful parameters, which indicate the IR and UV scales are related through some common birth. SUSY can be thought of as a symmetry-motivated example that generates a natural relation of the former type, while scale-invariant UV completions provide symmetry-motivated examples of the latter type.

\footnotetext{
${ }^{8}$ Recent works have focused on threshold effects, assuming the quadratic divergences are dealt with by an as-yet unknown mechanism [7, 9] (the so-called "miraculous cancellation" [8]). Scale-invariance provides a symmetry-based rationale for the neglect of quadratic divergences, offering a motivation for this perspective.
} 


\section{Acknowledgments}

The authors thank R. Foot and R. Volkas. This work was supported in part by the Australian Research Council.

Open Access. This article is distributed under the terms of the Creative Commons Attribution License (CC-BY 4.0), which permits any use, distribution and reproduction in any medium, provided the original author(s) and source are credited.

\section{References}

[1] K.G. Wilson, The Renormalization Group and Strong Interactions, Phys. Rev. D 3 (1971) 1818 [inSPIRE].

[2] L. Susskind, Dynamics of Spontaneous Symmetry Breaking in the Weinberg-Salam Theory, Phys. Rev. D 20 (1979) 2619 [inSPIRE].

[3] S. Weinberg, Phenomenological Lagrangians, Physica A 96 (1979) 327 [InSPIRE].

[4] S. Dubovsky, V. Gorbenko and M. Mirbabayi, Natural Tuning: Towards A Proof of Concept, JHEP 09 (2013) 045 [arXiv: 1305.6939] [INSPIRE].

[5] R. Barbieri, Electroweak theory after the first Large Hadron Collider phase, Phys. Scripta T 158 (2013) 014006 [arXiv:1309.3473] [INSPIRE].

[6] A. de Gouvêa, D. Hernández and T.M.P. Tait, Criteria for Natural Hierarchies, Phys. Rev. D 89 (2014) 115005 [arXiv: 1402.2658] [InSPIRE].

[7] M. Farina, D. Pappadopulo and A. Strumia, A modified naturalness principle and its experimental tests, JHEP 08 (2013) 022 [arXiv: 1303.7244] [INSPIRE].

[8] G.F. Giudice, Naturalness after LHC8, PoS (EPS-HEP 2013) 163 [arXiv:1307.7879] [INSPIRE].

[9] R. Foot, A. Kobakhidze, K.L. McDonald and R.R. Volkas, Poincaré Protection for a Natural Electroweak Scale, Phys. Rev. D 89 (2014) 115018 [arXiv:1310.0223] [inSPIRE].

[10] W.A. Bardeen, On naturalness in the standard model, FERMILAB-CONF-95-391 [INSPIRE].

[11] C. Wetterich, Fine Tuning Problem and the Renormalization Group, Phys. Lett. B 140 (1984) 215 [inSPIRE].

[12] C. Wetterich, The Mass Of The Higgs Particle, DESY-87-154 [InSPIRE].

[13] F. Vissani, Do experiments suggest a hierarchy problem?, Phys. Rev. D 57 (1998) 7027 [hep-ph/9709409] [INSPIRE].

[14] J.A. Casas, J.R. Espinosa and I. Hidalgo, Implications for new physics from fine-tuning arguments. 1. Application to SUSY and seesaw cases, JHEP 11 (2004) 057 [hep-ph/0410298] [INSPIRE].

[15] J.A. Casas, J.R. Espinosa and I. Hidalgo, Expectations for LHC from naturalness: modified versus SM Higgs sector, Nucl. Phys. B 777 (2007) 226 [hep-ph/0607279] [INSPIRE].

[16] R.R. Volkas, A.J. Davies and G.C. Joshi, Naturalness Of The Invisible Axion Model, Phys. Lett. B 215 (1988) 133 [INSPIRE]. 
[17] M.J.G. Veltman, The Infrared-Ultraviolet Connection, Acta Phys. Polon. B 12 (1981) 437 [INSPIRE].

[18] I. Jack and D.R.T. Jones, Naturalness Without Supersymmetry?, Phys. Lett. B 234 (1990) 321 [inSPIRE].

[19] M.S. Al-sarhi, I. Jack and D.R.T. Jones, Quadratic divergences in gauge theories, Z. Phys. C 55 (1992) 283 [inSPIRE].

[20] M. Chaichian, R. Gonzalez Felipe and K. Huitu, On quadratic divergences and the Higgs mass, Phys. Lett. B 363 (1995) 101 [hep-ph/9509223] [INSPIRE].

[21] Y. Hamada, H. Kawai and K.-y. Oda, Bare Higgs mass at Planck scale, Phys. Rev. D 87 (2013) 053009 [arXiv:1210.2538] [InSPIRE].

[22] F. Jegerlehner, The Standard model as a low-energy effective theory: what is triggering the Higgs mechanism?, Acta Phys. Polon. B 45 (2014) 1167 [arXiv: 1304.7813] [InSPIRE].

[23] F. Jegerlehner, The hierarchy problem of the electroweak Standard Model revisited, arXiv:1305.6652 [INSPIRE].

[24] I. Masina and M. Quirós, On the Veltman Condition, the Hierarchy Problem and High-Scale Supersymmetry, Phys. Rev. D 88 (2013) 093003 [arXiv: 1308.1242] [INSPIRE].

[25] O. Antipin, M. Mojaza and F. Sannino, Conformal Extensions of the Standard Model with Veltman Conditions, Phys. Rev. D 89 (2014) 085015 [arXiv: 1310.0957] [INSPIRE].

[26] M.T. Grisaru, W. Siegel and M. Roček, Improved Methods for Supergraphs, Nucl. Phys. B 159 (1979) 429 [inSPIRE].

[27] S.R. Coleman and E.J. Weinberg, Radiative Corrections as the Origin of Spontaneous Symmetry Breaking, Phys. Rev. D 7 (1973) 1888 [INSPIRE].

[28] A. Kobakhidze, Scale invariance and the electroweak symmetry breaking, PoS (ICHEP2012) 156 [arXiv: 1303.5897] [INSPIRE].

[29] K.A. Meissner and H. Nicolai, Conformal Symmetry and the Standard Model, Phys. Lett. B 648 (2007) 312 [hep-th/0612165] [INSPIRE].

[30] R. Foot, A. Kobakhidze and R.R. Volkas, Electroweak Higgs as a pseudo-Goldstone boson of broken scale invariance, Phys. Lett. B 655 (2007) 156 [arXiv:0704.1165] [INSPIRE].

[31] R. Foot, A. Kobakhidze, K.L. McDonald and R.R. Volkas, Neutrino mass in radiatively-broken scale-invariant models, Phys. Rev. D 76 (2007) 075014 [arXiv:0706.1829] [INSPIRE].

[32] R. Foot, A. Kobakhidze, K.L. McDonald and R.R. Volkas, A Solution to the hierarchy problem from an almost decoupled hidden sector within a classically scale invariant theory, Phys. Rev. D 77 (2008) 035006 [arXiv: 0709.2750] [INSPIRE].

[33] R. Foot, A. Kobakhidze and R.R. Volkas, Stable mass hierarchies and dark matter from hidden sectors in the scale-invariant standard model, Phys. Rev. D 82 (2010) 035005 [arXiv: 1006.0131] [INSPIRE].

[34] S. Iso, N. Okada and Y. Orikasa, Classically conformal B - L extended Standard Model, Phys. Lett. B 676 (2009) 81 [arXiv:0902 .4050] [INSPIRE].

[35] S. Iso, N. Okada and Y. Orikasa, The minimal B $-L$ model naturally realized at TeV scale, Phys. Rev. D 80 (2009) 115007 [arXiv: 0909.0128] [INSPIRE]. 
[36] M. Holthausen, M. Lindner and M.A. Schmidt, Radiative Symmetry Breaking of the Minimal Left-Right Symmetric Model, Phys. Rev. D 82 (2010) 055002 [arXiv:0911.0710] [InSPIRE].

[37] T. Hur and P. Ko, Scale invariant extension of the standard model with strongly interacting hidden sector, Phys. Rev. Lett. 106 (2011) 141802 [arXiv:1103.2571] [INSPIRE].

[38] L. Alexander-Nunneley and A. Pilaftsis, The Minimal Scale Invariant Extension of the Standard Model, JHEP 09 (2010) 021 [arXiv:1006.5916] [INSPIRE].

[39] R. Foot, A. Kobakhidze and R.R. Volkas, Cosmological constant in scale-invariant theories, Phys. Rev. D 84 (2011) 075010 [arXiv: 1012.4848] [INSPIRE].

[40] R. Foot and A. Kobakhidze, Electroweak Scale Invariant Models with Small Cosmological Constant, arXiv:1112.0607 [INSPIRE].

[41] K. Ishiwata, Dark Matter in Classically Scale-Invariant Two Singlets Standard Model, Phys. Lett. B 710 (2012) 134 [arXiv:1112.2696] [INSPIRE].

[42] J.S. Lee and A. Pilaftsis, Radiative Corrections to Scalar Masses and Mixing in a Scale Invariant Two Higgs Doublet Model, Phys. Rev. D 86 (2012) 035004 [arXiv:1201.4891] [INSPIRE].

[43] N. Okada and Y. Orikasa, Dark matter in the classically conformal B - L model, Phys. Rev. D 85 (2012) 115006 [arXiv:1202.1405] [inSPIRE].

[44] S. Iso and Y. Orikasa, TeV Scale B - L model with a flat Higgs potential at the Planck scale: In view of the hierarchy problem, Prog. Theor. Exp. Phys. 2013 (2013) 023B08 [arXiv: 1210.2848] [INSPIRE].

[45] C. Englert, J. Jaeckel, V.V. Khoze and M. Spannowsky, Emergence of the Electroweak Scale through the Higgs Portal, JHEP 04 (2013) 060 [arXiv:1301.4224] [INSPIRE].

[46] M. Heikinheimo, A. Racioppi, M. Raidal, C. Spethmann and K. Tuominen, Physical Naturalness and Dynamical Breaking of Classical Scale Invariance, Mod. Phys. Lett. A 29 (2014) 1450077 [arXiv:1304.7006] [InSPIRE].

[47] M. Heikinheimo, A. Racioppi, M. Raidal, C. Spethmann and K. Tuominen, Dark Supersymmetry, Nucl. Phys. B 876 (2013) 201 [arXiv:1305.4182] [InSPIRE].

[48] T. Hambye and A. Strumia, Dynamical generation of the weak and Dark Matter scale, Phys. Rev. D 88 (2013) 055022 [arXiv: 1306. 2329] [INSPIRE].

[49] I. Bars, P. Steinhardt and N. Turok, Local Conformal Symmetry in Physics and Cosmology, Phys. Rev. D 89 (2014) 043515 [arXiv:1307.1848] [InSPIRE].

[50] M. Heikinheimo, A. Racioppi, M. Raidal and C. Spethmann, Twin Peak Higgs, Phys. Lett. B 726 (2013) 781 [arXiv:1307.7146] [InSPIRE].

[51] C.D. Carone and R. Ramos, Classical scale-invariance, the electroweak scale and vector dark matter, Phys. Rev. D 88 (2013) 055020 [arXiv:1307.8428] [INSPIRE].

[52] G. Marques Tavares, M. Schmaltz and W. Skiba, Higgs mass naturalness and scale invariance in the UV, Phys. Rev. D 89 (2014) 015009 [arXiv: 1308.0025] [INSPIRE].

[53] A. Farzinnia, H.-J. He and J. Ren, Natural Electroweak Symmetry Breaking from Scale Invariant Higgs Mechanism, Phys. Lett. B 727 (2013) 141 [arXiv: 1308.0295] [INSPIRE].

[54] Y. Kawamura, Naturalness, Conformal Symmetry and Duality, Prog. Theor. Exp. Phys. 2013 (2013) 113B04 [arXiv:1308.5069] [InSPIRE]. 
[55] V.V. Khoze, Inflation and Dark Matter in the Higgs Portal of Classically Scale Invariant Standard Model, JHEP 11 (2013) 215 [arXiv: 1308.6338] [INSPIRE].

[56] E. Gabrielli, M. Heikinheimo, K. Kannike, A. Racioppi, M. Raidal and C. Spethmann, Towards Completing the Standard Model: Vacuum Stability, EWSB and Dark Matter, Phys. Rev. D 89 (2014) 015017 [arXiv: 1309.6632] [InSPIRE].

[57] T.G. Steele, Z.-W. Wang, D. Contreras and R.B. Mann, Viable dark matter via radiative symmetry breaking in a scalar singlet Higgs portal extension of the standard model, Phys. Rev. Lett. 112 (2014) 171602 [arXiv:1310.1960] [INSPIRE].

[58] M. Hashimoto, S. Iso and Y. Orikasa, Radiative symmetry breaking at the Fermi scale and flat potential at the Planck scale, Phys. Rev. D 89 (2014) 016019 [arXiv: 1310.4304] [InSPIRE].

[59] M. Holthausen, J. Kubo, K.S. Lim and M. Lindner, Electroweak and Conformal Symmetry Breaking by a Strongly Coupled Hidden Sector, JHEP 12 (2013) 076 [arXiv:1310.4423] [INSPIRE].

[60] S. Abel and A. Mariotti, Novel Higgs Potentials from Gauge Mediation of Exact Scale Breaking, Phys. Rev. D 89 (2014) 125018 [arXiv:1312.5335] [INSPIRE].

[61] C.T. Hill, Is the Higgs Boson Associated with Coleman-Weinberg Dynamical Symmetry Breaking?, Phys. Rev. D 89 (2014) 073003 [arXiv:1401.4185] [INSPIRE].

[62] J. Guo and Z. Kang, Higgs Naturalness and Dark Matter Stability by Scale Invariance, arXiv: 1401.5609 [INSPIRE].

[63] M. Hashimoto, S. Iso and Y. Orikasa, Radiative Symmetry Breaking from Flat Potential in various U(1)' models, Phys. Rev. D 89 (2014) 056010 [arXiv: 1401.5944] [InSPIRE].

[64] S. Benić and B. Radovčić, Electroweak breaking and Dark Matter from the common scale, Phys. Lett. B 732 (2014) 91 [arXiv: 1401.8183] [INSPIRE].

[65] A. Salvio and A. Strumia, Agravity, JHEP 06 (2014) 080 [arXiv:1403.4226] [INSPIRE].

[66] J. Kubo, K.S. Lim and M. Lindner, Electroweak Symmetry Breaking by QCD, arXiv: 1403.4262 [INSPIRE].

[67] V.V. Khoze, C. McCabe and G. Ro, Higgs vacuum stability from the dark matter portal, arXiv: 1403.4953 [INSPIRE].

[68] G.C. Dorsch, S.J. Huber and J.M. No, Cosmological Signatures of a UV-Conformal Standard Model, arXiv: 1403.5583 [INSPIRE].

[69] M. Shaposhnikov and D. Zenhausern, Quantum scale invariance, cosmological constant and hierarchy problem, Phys. Lett. B 671 (2009) 162 [arXiv:0809.3406] [INSPIRE].

[70] F. Gretsch and A. Monin, Dilaton: Saving Conformal Symmetry, arXiv:1308.3863 [INSPIRE].

[71] C. Tamarit, Running couplings with a vanishing scale anomaly, JHEP 12 (2013) 098 [arXiv: 1309.0913] [INSPIRE].

[72] K.A. Meissner and H. Nicolai, Effective action, conformal anomaly and the issue of quadratic divergences, Phys. Lett. B 660 (2008) 260 [arXiv:0710.2840] [INSPIRE].

[73] H. Aoki and S. Iso, Revisiting the Naturalness Problem: Who is afraid of quadratic divergences?, Phys. Rev. D 86 (2012) 013001 [arXiv:1201.0857] [INSPIRE]. 